\title{
Evaluating the significance of hypoglycemia in promoting insulin induced hypertension by using a glucose clamp model
}

\author{
Priyanka Prathipati $^{1 *}$, Debra W. Jackson ${ }^{2}$, Keith E. Jackson ${ }^{1}$ \\ ${ }^{1}$ Department of Basic Pharmaceutical Sciences, College of Pharmacy, University of Louisiana at Monroe, Monroe, LA 71201 \\ ${ }^{2}$ Division of Natural Sciences and Math, School of Health Sciences, Natural Sciences and Math, \\ Louisiana Delta Community College, Monroe, LA 71203. USA \\ *Corresponding author E-mail: kjackson@ulm.edu
}

\begin{abstract}
Background: Hypoglycemia unawareness is a detrimental risk factor in diabetic patients leading to serious complications if left untreated. Previous studies from our group determined that recurrent insulin induced hypoglycemia (RIIH) promotes hypertension.

Objective: We hypothesize that RIIH not insulin by itself is the source of hypertension and end organ damage in diabetic patients.

Methods: Male Sprague-Dawley-rats $(200-250 \mathrm{~g}, \mathrm{n}=18)$ were provided with glucose food $(125 \mathrm{~g}$ glucose/kg body weight) and glucose water $(0.1 \mathrm{~g}$ glucose $/ 100 \mathrm{~g}$ body weight $/ \mathrm{ml})$. They were treated with subcutaneous insulin injections $(7 \mathrm{U} / \mathrm{Kg})$ and blood glucose was monitored intermittently. Daily blood pressure was measured using the tail cuff method. Interstitial samples of ATP and angiotensinII (AngII) were collected by renal microdialysis and analyzed using luciferin-luciferase bioluminescent assay and EIA respectively. Reactive oxygen and nitrogen species in hearts and kidneys were analyzed using Electron Paramagnetic Resonance Spectroscopy (EPR).

Results: Renal interstitial ATP levels increased from $90.2 \pm 4.7 \mathrm{ng} / \mu 1$ to $99.6 \pm 8.7 \mathrm{ng} / \mu 1$ (not significant) and AngII from $0.15 \pm 0.02 \mathrm{ng} / \mathrm{ml}$ to $0.13 \pm 0.05 \mathrm{ng} / \mathrm{ml}$ (not significant) from day 0 to 14 . There was no significant change in mean arterial pressure $(121.3 \pm 1.4 \mathrm{mmHg}$ on day 0 to $127.8 \pm 1.4 \mathrm{mmHg}$ on day 14). Oxidative stress was reduced compared to RIIH model, which was evident from the EPR spectra. Conclusion: We demonstrated that hypertension induced end organ damage in diabetics is due to insulin induced hypoglycemia not insulin alone (by itself).
\end{abstract}

Keywords: Angiotensin II; ATP; Diabetes; Hypertension; Microdialysis.

\section{Introduction}

Diabetes is characterized by increased blood-glucose levels due to defects in insulin production, insulin action or both, and often leads to various complications. Major complications of diabetes include hypoglycemia, diabetic coma, diabetic retinopathy, diabetic neuropathy, diabetic nephropathy, diabetic cardiomyopathy, hypertension, and amputations. Diabetes is projected to be the seventh leading cause of death by 2030 according to World Health Organization (WHO)(Mathers\& Loncar, 2006). Centers for Disease Control and Prevention (CDC) reported that $71 \%$ of diabetic patients (age $\geq 18$ years) were hypertensive during 2009-2012. In 2011, diabetes was found to be the primary cause of kidney failure in $44 \%$ of the patients (http://www.cdc.gov/diabetes/pubs/statsreport14/national-diabetes-report-web.pdf ).

Increasing prevalence of diabetes mellitus during the past decade has dramatically increased the incidence of end-stage renal disease (ESRD). Important contributors of ESRD include hypertension and hyperinsulinemia, which frequently coexist(El-Atat et al., 2004). Furthermore, hypertension was found to be associated with hyperinsulinemia through several mechanisms, one of which is tissue AngII and aldosterone actions, leading to vascular resistance(El-Atat et al., 2004). Hyperinsulinemia causes hypertension by increasing sodium retention and stimulating sympathetic activity. It also reduces the release of nitric oxide (NO) thus aggravating the condition and promotes endothelial dysfunction (Purohit \& Mathur,
2013; P. Prathipati et al., 2014). Circulating AngII has been reported to be elevated in many diabetic models(Eadington et al., 1994; Harrison-Bernard et al., 2002).Previous studies from our laboratory demonstrated the role of AngII in elevating HO-1, which in turn increased carbonmonoxide (CO) levels and ultimately increased mean arterial pressure (MAP) (S. Quadri et al., 2013; S. Quadri et al., 2014b). We also demonstrated that recurrent insulin induced hypoglycemia (RIIH) elevates MAP by enhancing circulating AngII. There was a dose-dependent decrease in blood-glucose levels with insulin treatment, which caused an elevation in blood pressure(S. Quadri et al., 2014a)due to an elevation in interstitial AngII and adenosine triphosphate (ATP) levels (P. Prathipati et al., 2015). ATP is not only the energy currency of the cell, but also regulates various hemodynamic functions in cardiovascular tissues and organs like the kidney (Nishiyama et al., 2006; P. Prathipati et al., 2015).

Along with hypertension and other complications, diabetic patients often experience hypoglycemic episodes as they strive to attain normal blood-glucose levels. Hypoglycemia is a condition when plasma glucose levels are $<70 \mathrm{mg} / \mathrm{dl}(3.9 \mathrm{mmol} / \mathrm{L})$ (Davis et al., 1997). Hypoglycemia unawareness (HU) occurs when the system fails to recognize the fall in blood glucose below normal levels(Moghissi et al., 2013)or is the onset of neuroglycopenia before the occurrence of autonomic warning symptoms(de Galan et al., 2006). The risk of severe hypoglycemia is higher in the presence of HU (six-fold for type 1 diabetes mellitus (TIDM)(Geddes et al., 2008)and 17-fold for type 2 diabetes mellitus (T2DM)(Schopman et al., 2010). Iatrogenic hypoglycemia is due to interplay of absolute 
or relative insulin excess and compromised glucose counter regulation in type 1 and advanced type 2 diabetes. HU was observed in $40 \%$ of TIDM while severe hypoglycemia resulted in a $10 \%$ mortality rate in T2DM patients(Martin-Timon \& Del Canizo-Gomez, 2015). Severe hypoglycemia or HU resulted in increased risk of mortality in clinical trial studies like Action to Control Cardiovascular Risk in Diabetes (ACCORD) and the Action in Diabetes and Vascular Disease (ADVANCE) (Action to Control Cardiovascular Risk in Diabetes Study et al., 2008; Zoungas et al., 2010). However, the source of hypertension during insulin treatment still remains unresolved. Having observed the role of hyperinsulinemia on hypertension and the adverse effects of hypoglycemia in diabetic patients, the present study focuses on the effects of euglycemic hyperinsulinemia, wherein glucose levels are clamped during insulin treatment by providing excess glucose to compensate for recurrent insulin induced hypoglycemia. This study focuses on identifying whether the source of hypertension is insulin alone, or if it is due to hypoglycemia caused by recurrent insulin injections. The effects of euglycemia on renal-interstitial ATP, AngII and oxidative stress were studied by maintaining normal glucose levels during insulin treatment.

\section{Materials and methods}

\subsection{Materials}

CMA 30 linear microdialysis probes were purchased from CMA microdialysis (Harvard Apparatus, Holliston, MA), ATP bioluminescent assay kit, $\mathrm{D}(+)$ glucose, and deferoxamine mesylate were purchased from Sigma-Aldrich (St. Louis, MO), AngII EIA kits were from Phoenix Pharmaceuticals, Inc.,(Burlingame, CA) CPH and diethyldithiocarbamic acid(DETC) were purchased from Enzo Life Sciences (Farmingdale, NY). Isoflurane was obtained from Piramal Critical Care (Bethlehem, PA). Bruker EMX EPR spectrometer with $\mathrm{Q}$ microwave cavity was used.

\subsection{Animals}

Seven-week-old male Sprague-Dawley rats weighing between 200 and $250 \mathrm{~g}(\mathrm{n}=18)$ were housed at room temperature with $12 / 12$ hours light/dark cycle. They had free access to food and water throughout the experiment. All animal experiments were approved by the University of Louisiana at Monroe Institutional Animal Care and Use Committee (IACUC).

\subsection{Experimental procedure}

Animals were divided into two groups. A subset of 10 was used for blood pressure measurement by tail cuff method while the other subset of 8 was used for microdialysis experiments.

Animals were treated with a daily 7 Units $/ \mathrm{kg}$ subcutaneous dose of insulin for 14 days. This insulin dose was determined in previous studies to promote sustained hypoglycemia(S. Quadri et al., 2014a). Rats were fed glucose food (125g glucose $/ \mathrm{kg}$ bodyweight) and glucose water $(0.1 \mathrm{~g}$ glucose $/ 100 \mathrm{~g}$ bodyweight $/ \mathrm{ml})$ to maintain euglycemic conditions. One hour post insulin injections, blood pressure was measured daily by tail-cuff analysis (Model SC-1000 MSP, Hatteras, Cary, NC). Blood-glucose levels were monitored on day 0 (baseline), day 4 (early), day 8 (mid), and day 14 (late) using blood-glucose test strips. Animal weights, food intake and water intake were monitored daily.

Microdialysis surgery was performed according to previously described methods(P. Prathipati et al., 2012). Briefly, rats were anesthetized throughout the surgical procedure using isoflurane anesthesia setup (Ez-anesthesia system). A small midsagittal incision was made to excise the kidney. A microdialysis probe was inserted into the cortex of the kidney which was exited from the nape of the neck. This was attached to the microdialysis setup and infused with physiological saline $(3 \mu \mathrm{l} / \mathrm{min})$ for sample collection(Hoar \& Hickman, 1975). The incisions were sutured and animals were given 2-3 days to recover post-surgery. At the end of the experiment, kidneys were collected and the location of the microdialysis membrane was confirmed by surgical exposure of the probe. After the recovery period, animals were fed on glucose food (125 g glucose $/ \mathrm{kg}$ bodyweight $)$ and glucose water $(0.1 \mathrm{~g}$ glucose $/ 100 \mathrm{~g}$ bodyweight $/ \mathrm{ml}$ ). They were treated with a daily 7 Units $/ \mathrm{kg}$ dose of insulin (subcutaneous) for 14 days. Samples (interstitial fluid) were collected 1 hour post insulin injection for a period of 9 hours. Blood glucose was monitored intermittently. Collected samples were stored in $-80^{\circ} \mathrm{C}$ until analyzed.

On the $14^{\text {th }}$ day, rats were anesthetized with a single injection of thiobutabarbital sodium (Inactin, $120 \mathrm{mg} / \mathrm{kg}$ ip) and a tracheal tube was inserted to maintain an open airway. Catheters (PE-50 tubing filled with heparinized saline) were implanted into the carotid artery to allow for continuous monitoring of MAP and heart rate (HR). The arterial catheter was connected to a pressure transducer (model TSD104A, Biopac Systems, Santa Barbara, CA). Following surgical procedures, rats were allowed to stabilize and blood pressure was measured after 1 hour

\subsection{ATP analysis}

Luciferin-luciferase bioluminescent assay was used to analyze for ATP in the interstitial samples. Bioluminescent light is produced when ATP reacts with luciferin, firefly luciferase and oxygen. The light emitted is proportional to the amount of ATP present.

\subsection{AngII analysis}

AngII EIA kit was used to measure AngII in the interstitial samples based on the principle of competitive enzyme immunoassay. The intensity of the yellow color produced due to the interaction of streptavidin-horse radish peroxidase (SA-HRP) is inversely proportional to the amount of peptide in the samples. The unknown concentration of the AngII in the samples was determined from the standard curve.

\subsection{EPR spectrometer}

Electron Paramagnetic Resonance Spectrometer (EPR) was used to analyze for the amount of reactive oxygen species(ROS) and peroxynitrite(ONOO-) present in the interstitial samples. Spin probe $\mathrm{CPH}$ (1-hydroxy-3-carboxy-2,2,5,5-tetramethylpyrrolidine. $\mathrm{HCl}$ ) was used for superoxide radical and ONOO-. As the half-life of the free radicals is very short, spin probes reacts and forms stable radicals. This increases the half-life of the free radicals to several hours and helps in stabilizing and quantifying them(Dikalov et al., 2007). Kidneys and hearts were harvested at the end of the experiment and flash frozen to store until analyzed. At the time of the experiment, kidneys and hearts were sliced into similar sized $2 \mathrm{~mm}$ samples and incubated for 1 hour at $37^{\circ} \mathrm{C}$ in $0.5 \mathrm{ml}$ of Krebs HEPES buffer(pH 7.4) containing $25 \mu \mathrm{M}$ deferoxamine mesylate and $5 \mu \mathrm{M}$ DETC along with 5mM of CPH(Elks et al., 2009). The following settings were used to record the EPR spectra: field sweep, 80G; microwave frequency, $9.39 \mathrm{GHz}$; microwave power, $2 \mathrm{~mW}$; modulation amplitude, 5G; conversion time, $327.68 \mathrm{~ms}$; time constant, $5242.88 \mathrm{~ms}$; 512 points resolution; and receiver gain, $1 \times 10^{4}$ (Dikalova et al., 2005). The amplitude of the signal was measured and quantitated to determine the amount of superoxide and ONOO- present in the samples.

\subsection{Statistics}

Data were expressed as mean $\pm \mathrm{SE}$ and analyzed by analysis of variance (ANOVA) followed by Tukey-Kramer multiple comparison test when appropriate (INSTAT 3$).(\mathrm{P}<0.05)$ was accepted as statistically significant. 


\section{Results}

\subsection{Blood glucose levels in glucose clamp animals after insulin treatment}

Blood glucose levels were monitored during days $0,4,8$ and 14 respectively. Glucose clamping was achieved during insulin injections by providing glucose in water and food, which maintained a euglycemic state. Consistent levels of glucose were found during the glucose clamp period without any hypoglycemic episodes (Fig.1). Blood glucose levels were found to be $95 \pm 5,89 \pm 5,89 \pm 0$ and $91 \pm 9 \mathrm{mg} / \mathrm{dL}$ on day $0,4,8$ and 14 respectively.

\section{Blood Glucose}

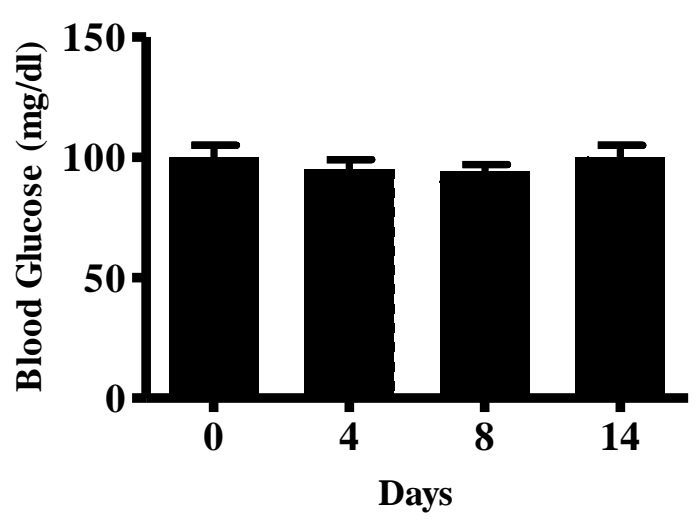

Figure 1: Blood glucose measurement in glucose fed animals. There was no significant drop in the blood glucose of the animals fed with glucose food and water during insulin treatment. Thus the euglycemic condition was achieved. Values are mean \pm SEM $(\mathrm{N}=18)$.

\subsection{Effect of glucose clamp on blood pressure}

In the awake animals, glucose supplementation attenuated the RIIH mediated increase in MAP when compared to animals treated with $7 \mathrm{U} / \mathrm{Kg}$ without external glucose supplement (Fig. 2) (S. Quadri et al., 2014a). There were no significant differences between the MAP of the animals. Systolic blood pressure was also maintained constant without any significant increases. Similarly diastolic pressure of animals supplemented with glucose did not show any significant change as compared to animals treated with $7 \mathrm{U} / \mathrm{Kg}$ alone.

\subsection{Effect of glucose clamp on renal interstitial ATP lev- els}

During the 2 week period, no significant changes in ATP levels were observed from day 0 which was $90.2 \pm 4.7 \mathrm{ng} / \mu$ l through day 14 which was $99.6 \pm 8.7 \mathrm{ng} / \mu \mathrm{l}$ (Fig.3 (a)). Hence glucose clamping attenuated the observed elevation in renal interstitial ATP levels thus preventing any disturbances in the tubuloglomerular feedback (TGF) mechanism.

\subsection{Effect of glucose clamp on renal interstitial Ang II levels}

During insulin treatment, no significant differences were observed in AngII concentrations from day 0 which was $0.15 \pm 0.02 \mathrm{ng} / \mathrm{ml}$ to day 14 which was $0.13 \pm 0.05 \mathrm{ng} / \mathrm{ml}$ (Fig.3 (b)).This data suggests that, the elevated levels of Ang II during hyperinsulinemia can be normalized by maintaining euglycemic conditions.

\subsection{Effect of glucose clamp on oxidative stress}

There was no significant change in ROS and RNS levels produced during the chronic treatment with $7 \mathrm{U} / \mathrm{Kg}$ insulin when euglycemic conditions were maintained. Kidney and hearts were incubated with CPH (Fig.4 (a,b)) producing EPR spectra and bar graphs (Fig.5) which indicate the presence of superoxide and peroxynitrite. Elevated oxidative stress during insulin induced hypoglycemic condition was previously reported(P. Prathipati et al., 2015).The present data suggests that, oxidative stress can be mitigated by maintaining normalized glucose levels during insulin treatment.

\section{Discussion}

The current study demonstrates that euglycemia attenuates hypertensive effects observed in diabetic conditions during insulin treatment. These results suggest that, hypoglycemia which is the result of recurrent insulin treatment is the cause of the observed hypertension in diabetic patients. In this study, hypoglycemia is neutralized by providing sufficient glucose to the animals to maintain euglycemia during insulin treatment. There was no significant difference in renal interstitial ATP or AngII levels, or in blood pressure. Our previous studies demonstrated that RIIH produced a significant elevation in ATP and AngII levels and eventual hypertension. Whereas we reported an elevation in renal interstitial ATP levels from day 8 which triggered renal AngII levels from day 10 during 14 days insulin treatment (S. Quadri et al., 2014a; P. Prathipati et al., 2015). The data together suggests that the observed hypertension can be attributed to hypoglycemia but not insulin alone. This indicates the adverse role of hypoglycemia during insulin treatment. RIIH promoting hypertension maybe neutralized by maintaining euglycemic conditions.

\section{Blood Glucose}

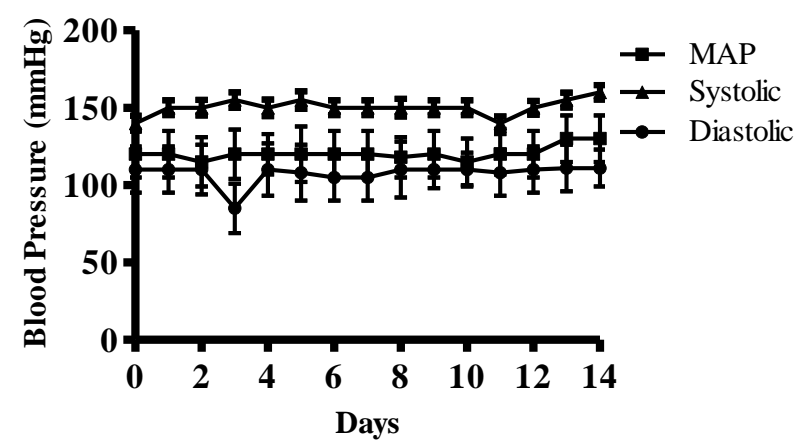

Figure 2: Effect of subcutaneous administration of insulin on blood pressure in awake animals. Animals were treated with subcutaneous injections of humulin and provided with glucose food. Mean arterial pressure (MAP), Systolic blood pressure (SBP) and Diastolic blood pressure (DBP), was monitored over a period of 2 weeks with tail cuff analysis post insulin injections. There was no significant difference observed. Values are mean \pm $\mathrm{SD}(\mathrm{N}=10)$.

A few clinical studies reported the beneficial approach of continuous glucose monitoring (CGM) in type I diabetic patients which reduced severe hypoglycemia (Maran et al., 2010; Choudhary et al., 2013; van Beers et al., 2015; Bally et al., 2016). However, the underlying mechanism remains to be elucidated. In the current study, the effects of hypoglycemia are identified by treating the animals with insulin and simultaneously maintaining euglycemic levels in the body to nullify the effects of insulin treatment. Thus, normal blood glucose levels in the body attenuated insulin induced hypertension which is evident from the blunted levels of renal interstitial ATP and Ang II levels and reduced blood pressure (Fig.2, 3). Previous studies have reported the role of ATP in maintaining the TGF mechanism by acting as a signaling component between macula densa cells and the afferent arteriole. This aides in regulating glomerular filtration rates to maintain renal health (Komlosi et al., 2005). It was also demonstrated that renal interstitial ATP regulates TGF dependent variations in renal vascular resistance (Nishiyama et al., 2000; Nishiyama et al., 2001; Nishiyama et al., 2006). Maintaining normal blood glucose levels might help in restoring the lost equilibrium of the TGF mechanism. This in turn would assist in 
regulating the renin angiotensin-aldosterone system (RAAS) by decreasing renal interstitial Ang II levels thus attenuated hypertension. The RAAS plays a crucial role in maintaining arterial blood pressure and in regulating physiologic and pathophysiologic processes in the kidney(Ruster\& Wolf, 2006). Emerging evidence has demonstrated an increase in mean arterial pressure with subcutaneous infusions of AngII (Kobori et al., 2001). The current study provides one physiological mechanism where maintenance of euglycemia improves the quality of life for diabetic patients. Exploring and understanding the roles of ATP and Ang II in regulating TGF and RAAS mechanisms suggests that more targeted therapeutic approaches could be employed over systemic blockade of Ang II receptors.

\section{Blood Glucose}

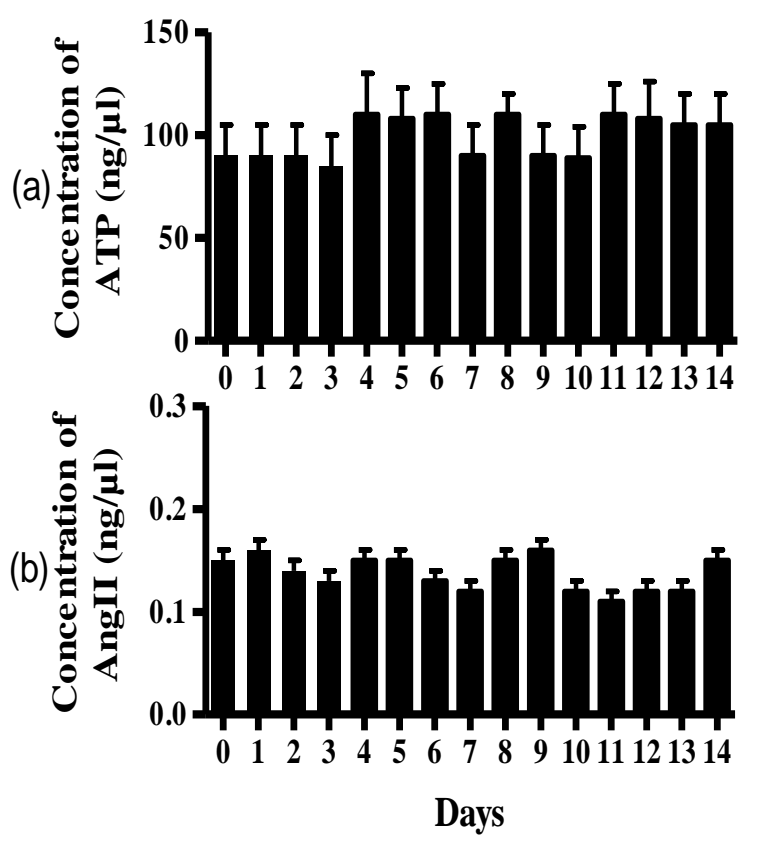

Figure 3: Analysis of ATP and Ang II. In awake rats, physiological saline was perfused through the microdialysis probes inserted in kidneys. Perfusate was collected post insulin (7Units/kg) injections continuously for $9 \mathrm{hr}$ during 2 weeks period while providing glucose diet. Samples were then analyzed for (a) ATP using luciferin-luciferase assay and (b) AngII using EIA There was no significant difference in either ATP or Ang II levels from day 0 through day 14 . Values are mean $\pm \operatorname{SEM}(\mathrm{N}=8)$.

The overlap between diabetes and hypertension increases the risk of ESRD. Hyperglycemia and hypertension can impair endothelial cells leading to oxidative stress. Increased levels of ROS were observed with subcutaneous infusion of AngII by stimulating NAD(P)H oxidase(Touyz \& Schiffrin, 1999). AngII induces renal damage either by vasoconstriction of the efferent arteriole or by autoregulation of the afferent arteriole (Baltatzi et al., 2011). It also stimulates renal inflammation and fibrosis contributing to kidney damage (Wolf et al., 2003). High intrarenal concentrations of AngII and reduced sodium excretion were observed in several experimental AngII dependent hypertensive models (Navar et al., 2002; Ruiz-Ortega et al., 2006; Kopkan et al., 2006). Several in vitro studies also reported the role of ATP in promoting oxidative stress (Chan et al., 1998; Sauer et al., 2001; Nishiyama et al., 2004). In the current study, the maintenance of normal blood glucose levels attenuated oxidative stress which may be due to reduced interstitial ATP and Ang II levels, when compared with that of insulin treatment alone (Fig. 4,5)(S. Quadri et al., 2014a; P. Prathipati et al., 2015). However, superoxide and peroxynitrite levels were higher in the heart as compared to the kidney (Fig. 4, 5). This remains as one of the interesting areas to understand the underlying mechanism of more cardiac ROS production compared to renal ROS levels and the interlink between cardio-renal complications. This study illustrates the importance of achieving near normal blood glucose levels in diabetic patients.

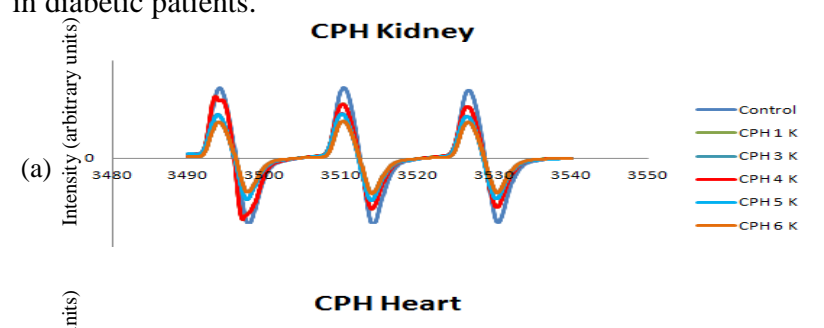

(b)

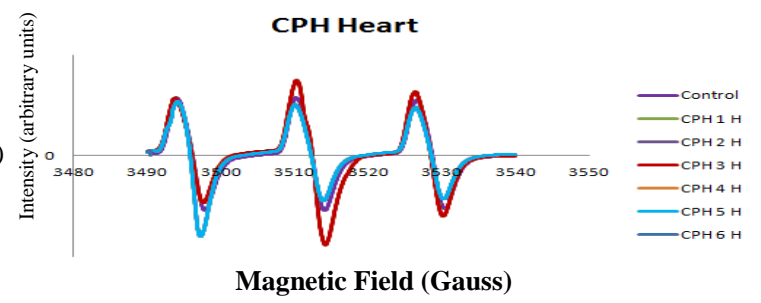

Figure 4: Detection of superoxide and peroxynitrite. EPR spectroscopy of (a) kidneys and (b) hearts collected on $14^{\text {th }}$ day of insulin treatment were incubated for $60 \mathrm{~min}$ with $\mathrm{CPH}$.

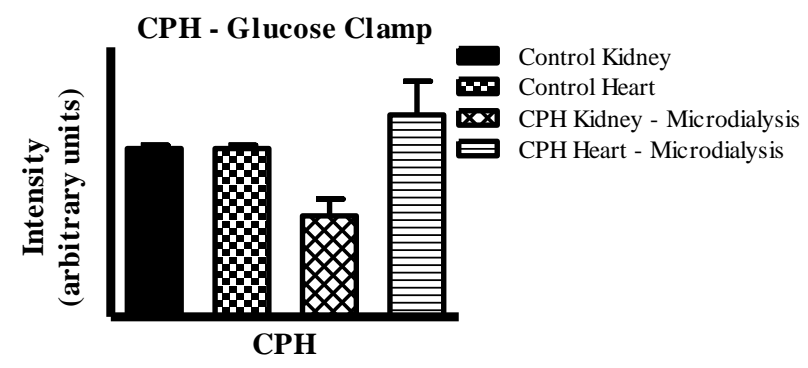

Figure 5: Detection of superoxide and peroxynitrite. Representative bar graphs indicate the oxidative stress produced in kidney and heart when treated with $\mathrm{CPH}$ to detect superoxide and peroxynitrite. Maintenance of euglycemia reduced the superoxide and peroxynitrite levels in kidney but not significantly when compared with control CPH. There was no significant change observed in superoxide and peroxynitrite production. Values are mean $\pm \operatorname{SEM}(\mathrm{N}=8)$.

\section{Conclusion}

In summary the present study demonstrates that euglycemia attenuates the elevated renal ATP which further blunts production of Ang II. This might restore the TGF and RAAS systems and helps in regulating normal blood pressure via a reduction in oxidative stress (Fig. 6). The novelty of this study is to establish an interrelationship between euglycemia, TGF and RAAS systems during recurrent insulin treatment. As the current study was conducted in healthy rats treated with insulin, additional studies in diabetic models are warranted to explore the role of these mechanisms in pathological conditions.

\section{Acknowledgment}

This work is supported by NIH-LBRN grant. The authors would like to sincerely thank Dr. Sharon Myers and her lab for assistance with the EPR studies.

\section{Conflict of Interests}

Authors confirm that there are no conflict of interest.

\section{References}

[1] Action to Control Cardiovascular Risk in Diabetes Study, G., Gerstein, H. C., Miller, M. E., Byington, R. P., Goff, D. C., Jr., Bigger, J. T., Friedewald, W. T. (2008). Effects of intensive glucose lowering 
in type 2 diabetes. $N$ Engl $J$ Med, 358(24), 2545-2559. https://doi.org/10.1056/NEJMoa0802743.

[2] Bally, L., Zueger, T., Pasi, N., Carlos, C., Paganini, D., \& Stettler, C. (2016). Accuracy of continuous glucose monitoring during differing exercise conditions. Diabetes Res Clin Pract, 112, 1-5. https://doi.org/10.1016/j.diabres.2015.11.012.

[3] Baltatzi, M., Savopoulos, C., \& Hatzitolios, A. (2011). Role of angiotensin converting enzyme inhibitors and angiotensin receptor blockers in hypertension of chronic kidney disease and renoprotection. Study results. Hippokratia, 15(Suppl 1), 27-32.

[4] Chan, C. M., Unwin, R. J., \& Burnstock, G. (1998). Potential functional roles of extracellular ATP in kidney and urinary tract. Exp Nephrol, 6(3), 200-207. https://doi.org/10.1159/000020524.

[5] Choudhary, P., Ramasamy, S., Green, L., Gallen, G., Pender, S. Brackenridge, A., Pickup, J. C. (2013). Real-time continuous glucose monitoring significantly reduces severe hypoglycemia in hypoglycemia-unaware patients with type 1 diabetes. Diabetes Care, 36(12), 4160-4162. https://doi.org/10.2337/dc13-0939.

[6] Davis, S. N., Shavers, C., Mosqueda-Garcia, R., \& Costa, F. (1997) Effects of differing antecedent hypoglycemia on subsequent counterregulation in normal humans. Diabetes, 46(8), 1328-1335. https://doi.org/10.2337/diab.46.8.1328.

[7] De Galan, B. E., Schouwenberg, B. J., Tack, C. J., \& Smits, P. (2006). Pathophysiology and management of recurrent hypoglycaemia and hypoglycaemia unawareness in diabetes. Neth J Med, 64(8), 269-279.

[8] Dikalov, S., Griendling, K. K., \& Harrison, D. G. (2007). Measurement of reactive oxygen species in cardiovascular studies. Hypertension, $\quad 49(4), \quad 717-727$ https://doi.org/10.1161/01.HYP.0000258594.87211.6b

[9] Dikalova, A., Clempus, R., Lassegue, B., Cheng, G., McCoy, J., Dikalov, S., Griendling, K. K. (2005). Nox1 overexpression potentiates angiotensin II-induced hypertension and vascular smooth muscle hypertrophy in transgenic mice. Circulation, 112(17), 2668-2676. https://doi.org/10.1161/CIRCULATIONAHA.105.538934.

[10] Eadington, D. W., Frier, B. M., \& Swainson, C. P. (1994). Renal tubular responses to low-dose infusion of angiotensin II in type 1 diabetes mellitus; relation to chronic glycaemic control. Nephrol Dial Transplant, 9(9), 1264-1270.

[11] El-Atat, F. A., Stas, S. N., McFarlane, S. I., \& Sowers, J. R. (2004) The relationship between hyperinsulinemia, hypertension and progressive renal disease. J Am Soc Nephrol, 15(11), 2816-2827. https://doi.org/10.1097/01.ASN.0000133698.80390.37.

[12] Elks, C. M., Mariappan, N., Haque, M., Guggilam, A., Majid, D. S., \& Francis, J. (2009). Chronic NF-\{kappa $\}$ B blockade reduces cytosolic and mitochondrial oxidative stress and attenuates renal injury and hypertension in SHR. Am J Physiol Renal Physiol, 296(2), F298305. https://doi.org/10.1152/ajprenal.90628.2008.

[13] Geddes, J., Schopman, J. E., Zammitt, N. N., \& Frier, B. M. (2008). Prevalence of impaired awareness of hypoglycaemia in adults with Type 1 diabetes. Diabet Med, 25(4), 501-504 https://doi.org/10.1111/j.1464-5491.2008.02413.x.

[14] Harrison-Bernard, L. M., Imig, J. D., \& Carmines, P. K. (2002). Renal AT1 receptor protein expression during the early stage of diabetes mellitus. Int $J$ Exp Diabetes Res, 3(2), 97-108. https://doi.org/10.1080/15604280214483.

[15] Hoar, W., \& Hickman, C. (1975). Ovariectomy and the estrous cycle of the rat-General and Comparitive Physiology.

[16] Kobori, H., Harrison-Bernard, L. M., \& Navar, L. G. (2001). Enhancement of angiotensinogen expression in angiotensin II-dependent hypertension. Hypertension, 37(5), 1329-1335 https://doi.org/10.1161/01.HYP.37.5.1329.

[17] Komlosi, P., Fintha, A., \& Bell, P. D. (2005). Renal cell-to-cell communication via extracellular ATP. Physiology (Bethesda), 20, 86-90. https://doi.org/10.1152/physiol.00002.2005.

[18] Kopkan, L., Castillo, A., Navar, L. G., \& Majid, D. S. (2006). Enhanced superoxide generation modulates renal function in ANG IIinduced hypertensive rats. Am J Physiol Renal Physiol, 290(1), F8086. https://doi.org/10.1152/ajprenal.00090.2005.

[19] Maran, A., Pavan, P., Bonsembiante, B., Brugin, E., Ermolao, A. Avogaro, A., \& Zaccaria, M. (2010). Continuous glucose monitoring reveals delayed nocturnal hypoglycemia after intermittent high-intensity exercise in nontrained patients with type 1 diabetes. Diabetes Technol Ther, 12(10), 763-768. https://doi.org/10.1089/dia.2010.0038.

[20] Martin-Timon, I., \& Del Canizo-Gomez, F. J. (2015). Mechanisms of hypoglycemia unawareness and implications in diabetic patients.

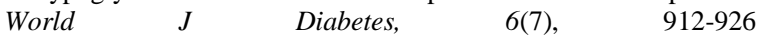
https://doi.org/10.4239/wjd.v6.i7.912.
[21] Mathers, C. D., \& Loncar, D. (2006). Projections of global mortality and burden of disease from 2002 to 2030. PLoS Med, 3(11), e442. https://doi.org/10.1371/journal.pmed.0030442.

[22] Moghissi, E., Ismail-Beigi, F., \& Devine, R. C. (2013). Hypoglycemia: minimizing its impact in type 2 diabetes. Endocr Pract, 19(3), 526-535. https://doi.org/10.4158/EP13005.RA.

[23] Navar, L. G., Harrison-Bernard, L. M., Nishiyama, A., \& Kobori, H. (2002). Regulation of intrarenal angiotensin II in hypertension. Hy$\left.\begin{array}{lllll}\text { pertension, } & 39(2 & \mathrm{Pt} & 2\end{array}\right), \quad 316-322$. https://doi.org/10.1161/hy0202.103821.

[24] Nishiyama, A., Jackson, K. E., Majid, D. S., Rahman, M., \& Navar, L. G. (2006). Renal interstitial fluid ATP responses to arterial pressure and tubuloglomerular feedback activation during calcium channel blockade. Am J Physiol Heart Circ Physiol, 290(2), H772-777. https://doi.org/10.1152/ajpheart.00242.2005.

[25] Nishiyama, A., Majid, D. S., Taher, K. A., Miyatake, A., \& Navar, L. G. (2000). Relation between renal interstitial ATP concentrations and autoregulation-mediated changes in renal vascular resistance. Circ Res, 86(6), 656-662. https://doi.org/10.1161/01.RES.86.6.656.

[26] Nishiyama, A., Majid, D. S., Walker, M., 3rd, Miyatake, A., \& Navar L. G. (2001). Renal interstitial atp responses to changes in arterial pressure during alterations in tubuloglomerular feedback activity. $\begin{array}{lllll}\text { Hypertension, } & 37(2 & \mathrm{Pt} & 2), & 753-759 .\end{array}$ https://doi.org/10.1161/01.HYP.37.2.753.

[27] Nishiyama, A., Rahman, M., \& Inscho, E. W. (2004). Role of interstitial ATP and adenosine in the regulation of renal hemodynamics and microvascular function. Hypertens Res, 27(11), 791-804. https://doi.org/10.1291/hypres.27.791.

[28] Prathipati, P., Alanazi, W., Fakhruddin, Jackson, D. W., \& Jackson, K. E. (2015). Role of interstitial ATP and angiotensin II in mediating renal injury induced by recurrent insulin induced hypoglycemia. $A n-$ nual Research and Review in Biology, 6(5), 9. https://doi.org/10.9734/ARRB/2015/16184.

[29] Prathipati, P., Quadri, S., Jackson, D. W., \& Jackson, K. E. (2012). Adaptation of Renal Microdialysis for chronic interstitial collection of Angiotensin II and ATP. International Journal Biology, 4(4). https://doi.org/10.5539/ijb.v4n4p127.

[30] Prathipati, P., Quadri, S., Jackson, D. W., \& Jackson, K. E. (2014) Role of nitric oxide in septal coronary arteries of obese zucker rats International Journal of Medicine, 2(1), 5 https://doi.org/10.14419/ijm.v2i1.1941.

[31] Purohit, P., \& Mathur, R. (2013). Hypertension association with serum lipoproteins, insulin, insulin resistance and C-Peptide: unexplored forte of cardiovascular risk in hypothyroidism. $N \mathrm{Am} \mathrm{J} \mathrm{Med}$ Sci, 5(3), 195-201. https://doi.org/10.4103/1947-2714.109187.

[32] Quadri, S., Prathipati, P., Jackson, D. W., \& Jackson, K. E. (2013). Augmentation of heme oxygenase Promotes acute angiotensin II induced hypertension Clinical and Experimental Medical Sciences, 1 , 22. https://doi.org/10.12988/cems.2013.13003.

[33] Quadri, S., Prathipati, P., Jackson, D. W., \& Jackson, K. E. (2014a). Haemodynamic consequences of recurrent insulin-induced hypoglycaemia. Clin Exp Pharmacol Physiol, 41(1), 81-88. https://doi.org/10.1111/1440-1681.12183.

[34] Quadri, S., Prathipati, P., Jackson, D. W., \& Jackson, K. E. (2014b) Inhibition of angiotensin II with captopril in management of hypertension induced by recurrent insulin induced hypoglycemia. International journal of medical sciences.

[35] Ruiz-Ortega, M., Esteban, V., Ruperez, M., Sanchez-Lopez, E., Rodriguez-Vita, J., Carvajal, G., \& Egido, J. (2006). Renal and vascular hypertension-induced inflammation: role of angiotensin II. Curr Opin Nephrol Hypertens, 15(2), 159-166. https://doi.org/10.1097/01.mnh.0000203190.34643.d4.

[36] Ruster, C., \& Wolf, G. (2006). Renin-angiotensin-aldosterone system and progression of renal disease. J Am Soc Nephrol, 17(11), 2985-2991. https://doi.org/10.1681/ASN.2006040356.

[37] Sauer, H., Klimm, B., Hescheler, J., \& Wartenberg, M. (2001). Activation of p90RSK and growth stimulation of multicellular tumor spheroids are dependent on reactive oxygen species generated after purinergic receptor stimulation by ATP. FASEB J, 15(13), 25392541. https://doi.org/10.1096/fj.01-0360fje.

[38] Schopman, J. E., Geddes, J., \& Frier, B. M. (2010). Prevalence of impaired awareness of hypoglycaemia and frequency of hypoglycaemia in insulin-treated type 2 diabetes. Diabetes Res Clin Pract, 87(1), 64-68. https://doi.org/10.1016/j.diabres.2009.10.013.

[39] Touyz, R. M., \& Schiffrin, E. L. (1999). Ang II-stimulated superoxide production is mediated via phospholipase $\mathrm{D}$ in human vascular smooth muscle cells. Hypertension, 34(4 Pt 2), 976-982. https://doi.org/10.1161/01.HYP.34.4.976.

[40] Van Beers, C. A., Kleijer, S. J., Serne, E. H., Geelhoed-Duijvestijn, P. H., Snoek, F. J., Kramer, M. H., \& Diamant, M. (2015). Design 
and rationale of the IN CONTROL trial: the effects of real-time continuous glucose monitoring on glycemia and quality of life in patients with type 1 diabetes mellitus and impaired awareness of hypoglycemia. BMC Endocr Disord, 15, 42. https://doi.org/10.1186/s12902015-0040-3.

[41] Wolf, G., Butzmann, U., \& Wenzel, U. O. (2003). The renin-angiotensin system and progression of renal disease: from hemodynamics to cell biology. Nephron Physiol, 93(1), P3-13. https://doi.org/10.1159/000066656.

[42] Zoungas, S., Patel, A., Chalmers, J., de Galan, B. E., Li, Q., Billot,

L., Group, A. C. (2010). Severe hypoglycemia and risks of vascular events and death. $N$ Engl J Med, 363(15), 1410-1418. https://doi.org/10.1056/NEJMoa1003795. 